\title{
Laboratorios virtuales web como herramienta de apoyo para prácticas de ingeniería no presenciales
}

\author{
Antonio González Sorribes \\ âUniversitat Politècnica de València, e-mail: angonsor@upvnet.upv.es
}

\begin{abstract}
This paper presents a method for development and implantation of virtual laboratories for control engineering subjects, conceived with the purpose of allowing and promoting non-attending laboratory practices. One of the remarkable avantages of the proposed method is the unique requirement for students to have access to a web browser, which implies more flexibility and low cost resources. This is due to the fact that such virtual labs are implemented in a standard HTML and JAVASCRIPT language programming, which are full compatible with commercial web browsers. Moreover, it is worthwhile mentioning that the freely available tool Easy Java Simulation (EJS) allows teachers to develop new virtual laboratories without being necessary to possess strong skills in advanced programming. Apart from reaching a higher degree of autonomy and flexibility, this method will allow improving the long-term quality of learning because of the possibility of integrating a great variety of different didactic examples, which can simulataneosuly be used in the classroom in combination with theoretical lessons.
\end{abstract}

Keywords: virtual laboratory, autonomy, long-term learning, flexibility, open educational resources, web browser, HTML, Javascript.

\section{Resumen}

Este trabajo presenta una metodología de desarrollo e implantación de laboratorios virtuales para asignaturas de ingeniería de control, concebidos con el propósito de permitir y favorecer la no presencialidad en prácticas de laboratorio. Una de las ventajas reseñables del método propuesto es que únicamente es necesario que los estudiantes dispongan de un navegador web, lo cual implica una mayor flexibilidad y un bajo coste en recursos. Esto es gracias al hecho de que los laboratorios virtuales están implementados en lenguaje estándar HTML y Javascript, totalmente compatible con los navegadores web comerciales. Cabe resaltar que la herramienta Easy Java Simulation (EJS) de libre disposición permite a los docentes desarrollar nuevos laboratorios virtuales sin necesidad de conocimientos avanzados de programación. Aparte de alcanzar un grado mayor de autonomía y flexibilidad, este método permitirá mejorar la percepción de la asignatura y la calidad de aprendizaje a largo plazo por la posibilidad que ofrece de integrar un amplio repertorio de ejemplos didácticos que pueden utilizarse en el aula de forma complementaria a los contenidos teóricos impartidos.

Palabras clave: laboratorio virtual, autonomía, aprendizaje a largo plazo, flexibilidad, recursos educativos abiertos, navegador web, HTML, Javascript. 


\section{Introducción}

En el aprendizaje de cualquier disciplina, y en especial dentro de la rama de la ingeniería, es fundamental que los estudiantes puedan contrastar los contenidos adquiridos a través de las clases teóricas. Esta es una de las principales ventajas, por ejemplo, de las denominadas metodologías activas (March, 2006), ya que permiten que los estudiantes puedan construir su propio conocimiento a partir del método de aprendizaje activo o "learning-by-doing" (Hackathorn, 2011). Este proceso permite alcanzar niveles cognitivos más complejos (Sosniak, 1994) según la taxonomía de Bloom (Bloom, 1956), mejorando significativamente la calidad del aprendizaje. No obstante, presenta la desventaja de que es generalmente más complicado de implementar cuando el número de estudiantes por grupo es elevado. Esto se debe principalmente a dos motivos: (1) La limitación temporal impuesta por el calendario académico en relación a la cantidad de contenidos a impartir y (2) La limitación de recursos hardware y software (ordenadores, maquetas, programas con licencia) para la realización de las prácticas.

Por el contrario, la impartición de la docencia a través del método tradicional de clases magistrales es el método más efectivo desde el punto de vista de cantidad de información transmitida por unidad de tiempo, lo que en cierto modo justifica su vigencia actual en el sistema educativo. No obstante, el inconveniente de este método es que "el alumno recibe la información de forma pasiva por parte del profesor y la interioriza de forma memorística" (Michel et al., 2009: 397).

Por tanto, uno de los constantes desafíos entre los docentes universitarios es la búsqueda de métodos más efectivos para conciliar los contenidos impartidos a través de las clases magistrales con la utilidad práctica percibida por el alumno de cara a su futura trayectoria profesional. Esto favorece el aprendizaje significativo establecido por el Dr. David Paul Ausubel basado en el principio de que "el ser humano aprende mucho más fácil todo aquello que tiene significado para él". No obstante, este reto se intensifica en materias con un elevado contenido teórico debido a que son generalmente recibidas con mayor grado de hostilidad por los estudiantes por la aparente falta de conexión entre los contenidos impartidos en el aula con la vertiente aplicada de la asignatura.

\subsection{Contexto académico}

El método propuesto se ha aplicado en la asignatura de Ingeniería de Control durante el primer cuatrimestre del curso académico 2019/2020, del tercer año del Grado en Electrónica Industrial del Campus de Teruel, en la Universidad de Zaragoza.

Tras recabar diferentes opiniones de alumnos en cursos anteriores, se ha observado que una gran mayoría señaló ciertas dificultades para conectar los contenidos teóricos adquiridos en el aula con la aplicación de ingeniería real. Esto se debe fundamentalmente a la densa carga conceptual y matemática inherente a los contenidos intrínsecos de este tipo de materias, que requiere por parte del alumo una gran capacidad de abstracción. Debido a estas características, muchos estudiantes pueden percibir la asignatura como tediosa y por consiguiente pueden llegar a perder la motivación y el interés.

Por tanto, otro desafío al que se enfrentan los docentes es motivar a los estudiantes en asignaturas de este perfil. Para ello es fundamental que los alumnos comprendan la utilidad de los conceptos y fórmulas que se les proporciona en el aula, siendo la forma más efectiva el uso de herramientas visuales donde puedan contrastar lo que previamente han aprendido. Esto es lo que tradicionalmente se lleva a cabo mediante la realización de prácticas de laboratorio, si bien existe una limitación que depende del número de maquetas y equipos disponibles. Además, la dedicación se restringe al horario de prácticas, ya que los alumnos no pueden disponer de dichos recursos fuera del laboratorio. 


\section{Objetivos}

Con la finalidad de mejorar la percepción de los estudiantes sobre este tipo de materias, promover la motivación intrínseca (Trenshaw, 2016) e incrementar el tiempo que los estudiantes pueden dedicar a la realización de las prácticas de la asignatura, surge la iniciativa de implementar laboratorios virtuales que pueden operar localmente en un computador personal.

El objectivo principal del trabajo es presentar el desarrollo de dos laboratorios virtuales compatibles con un navegador web para la asignatura de ingeniería de control. La primera propuesta consiste en el control de la posición angular de un aeropéndulo mediante un controlador proporcional integrador derivativo (PID), y la segunda consiste en el control de seguimiento de la trayectoria de una bola deslizante sobre un plano móvil.

\section{Desarrollo de la innovación}

En este apartado se describe el método seguido para llevar a cabo el desarrollo de las aplicaciones mediante las cuales los alumnos podrán realizar las prácticas de laboratorio.

En primer lugar, se describe la herramienta Easy Java Simulations (EJS) mediante la cual se puede desarrollar los laboratorios virtuales. En segundo lugar se describe los pasos seguidos para implementar un laboratorio virtual que permite el control de un aeropéndulo.

\subsection{Descripción de Easy Java Simulation (EJS)}

Easy Java Simulations (EJS) es una herramienta de desarrollo software escrita en código abierto, creada por docentes con el propósito de mejorar la enseñanza y el aprendizaje de las ciencias (Esquembre, 2004). EJS tiene la ventaja clave de que permite al usuario desarrollar laboratorios virtuales y remotos a través de un asistente dotado de un interfaz gráfico. Por lo tanto, no es necesario que el usuario de EJS tenga conocimientos avanzados en programación. Una vez creado un proyecto EJS, el motor interno de la aplicación permite compilar y generar un conjunto de ficheros HTML/Javascript (Flanagan, 2006) o Applets de JAVA (Hoff, 1995), además de otros archivos multimedia (imágenes, videos, etc) organizados en un directorio común. Una vez realizado este paso, se puede fácilmente distribuir el código a los estudiantes para que puedan realizar las prácticas únicamente abriendo el fichero HTML principal generado automáticamente por EJS.

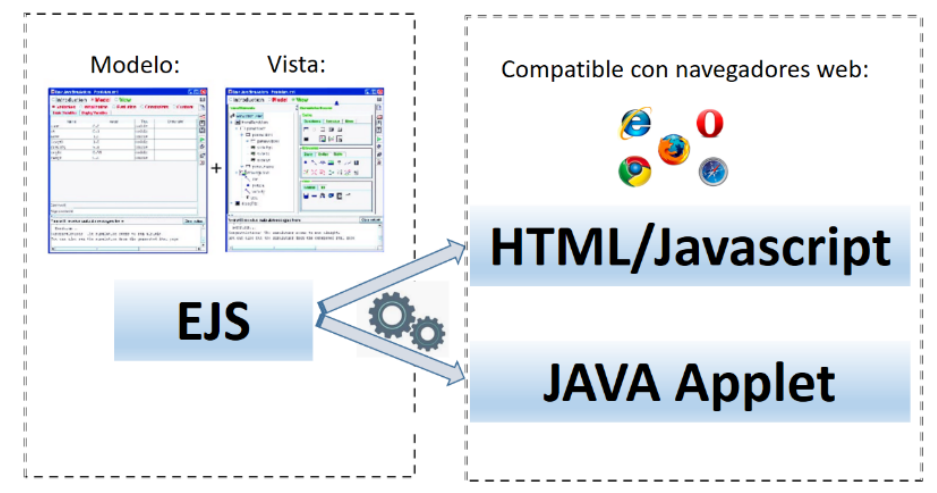

Fig. 1 Convertidor de código generado a partir de Easy Java Simulation a código HTML Javascript o Applets de JAVA 
No obstante, es recomendable exportar el proyecto a código HTML/Javascript ya que los navegadores web soportan este lenguaje de forma nativa, es decir, sin necesidad de utilizar complementos adicionales en el navegador que puedan dificultar su instalación y no garantizar el correcto desempeño funcional de la aplicación.

\subsection{EJS en el contexto educativo actual}

Actualmente, la herramienta EJS está siendo utilizada para generar laboratorios virtuales y remotos en ramas como la ingeniería de control (Chaos, 2013), robótica (Dormido, 2008), física (Christian, 2007), etc. con la finalidad de compartir recursos entre diferentes universidades. De este modo, se incrementa la variedad de aplicaciones para los estudiantes con coste mínimo. Además, el nivel actual de madurez en el desarrollo de laboratorios virtuales "permite su aplicación en otros contextos educativos más allá del ámbito universitario" (Heradio et al., 2016: 31). No obstante, a diferencia de los laboratorios virtuales, la implementación de los laboratorios remotos requiere del uso de algunas herramientas comerciales como MATLAB (López, 2002) o LABView (Johnson, 1997), y conlleva por tanto un mayor coste de implementación.

\subsection{Desarrollo de los laboratorios virtuales locales}

El laboratorio virtual se ejecutará únicamente en la aplicación front-end, es decir, mediante un navegador web que interpreta de forma nativa el lenguaje de marcas HTML y código Javascript. Cabe destacar que el laboratorio virtual se diseña para que sea operativo en modo local, es decir, eliminando la necesidad de tener conexión a internet durante la ejecución de la prácticas. Solo sería necesario disponer de conexión para poder descargar el código fuente de la aplicación del anillo digital docente, y para entregar el informe con los resultados para la evaluación. También cabe destacar que el proceso de instalación y puesta en funcionamiento del laboratorio virtual es inmediato independientemente de las características del sistema operativo de cada equipo en particular, siempre que dispongan de un navegador web.

\section{Resultados}

En este apartado se presentan dos propuestas de laboratorios virtuales desarrollados con EJS:

\subsection{Control de un aeropéndulo}

Un aeropéndulo consiste en una vara sujeta a un chasis por el extremo superior con libertad de movimiento, y en cuyo extremo inferior se coloca un propulsor con una hélice (Figura 2). El objetivo es controlar la posición angular $\theta$ del aeropéndulo mediante un algoritmo de control que proporciona el valor de tensión de alimentación que hay que proporcionar al motor que mueve el propulsor.

Una vez que los estudiantes disponen de esta aplicación, ellos podrán constratar el resultado de su diseño como si estuvieran trabajando físicamente con un aeropéndulo en un laboratorio. Es decir, a efectos meramente pedagógicos, los objetivos de aprendizaje se cumplen pero a menor coste. 


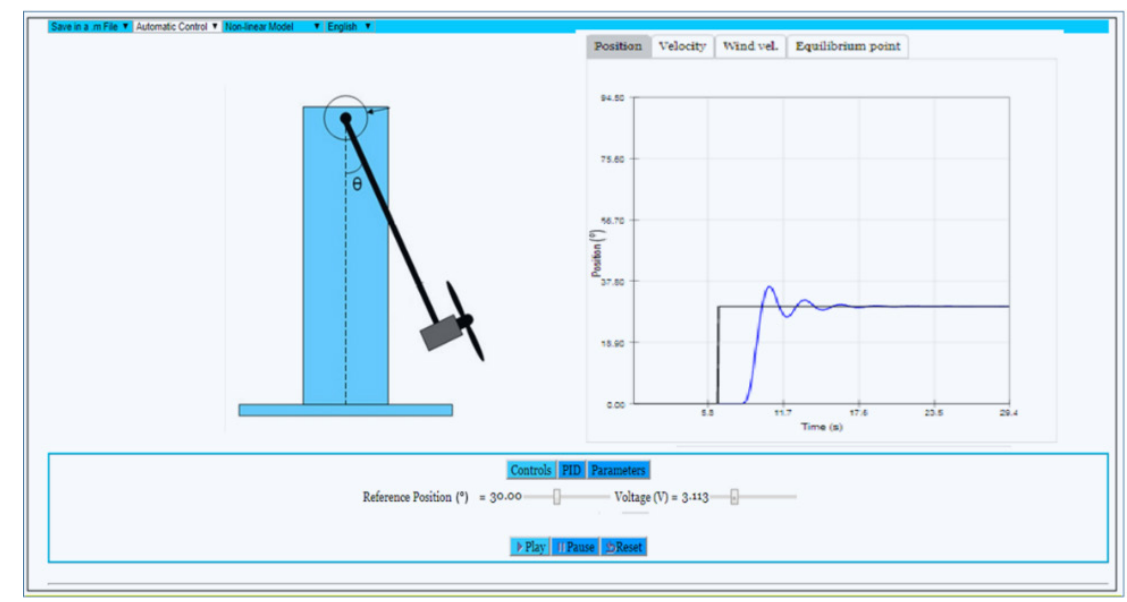

Fig. 2 Laboratorio virtual web para el control automático de la posición angular de un aeropéndulo

En la Figura 2 se muestra el interfaz de usuario que aparece tras abrir el fichero HTML con el navegador web. Mediante esta aplicación los estudiantes podrán visualizar el comportamiento del péndulo en función del diseño de controlador que hayan realizado, y cuyos parámetros podrán establecer en el panel inferior (botón PID). De este modo, los estudiantes podrán asociar con más facilidad por visualización directa los descriptores matemáticos que modelan la dinámica del sistema controlado con el comportamiento dinámico real del mismo.

Los ficheros HTML/Javascript se pondrán a disposición de los alumnos mediante su publicación dentro del anillo digital docente. De este modo, los alumnos solo tienen que descargarlos a un directorio local para poder realizar las prácticas.

\subsection{Control por visión de la posición de una bola en un plano móvil}

En el laboratorio virtual propuesto el estudiante deberá diseñar una estrategia de control PID para controlar la posición de una bola que descansa sobre un plano móvil cuyo movimiento está gobernado por dos motores (Figura 3). Por otro lado, se dispone de dos cámaras que permiten captar la posición de la bola sobre el plano aplicando algoritmos de procesado de imagen y posterior triangulación de la posición en tres dimensiones.

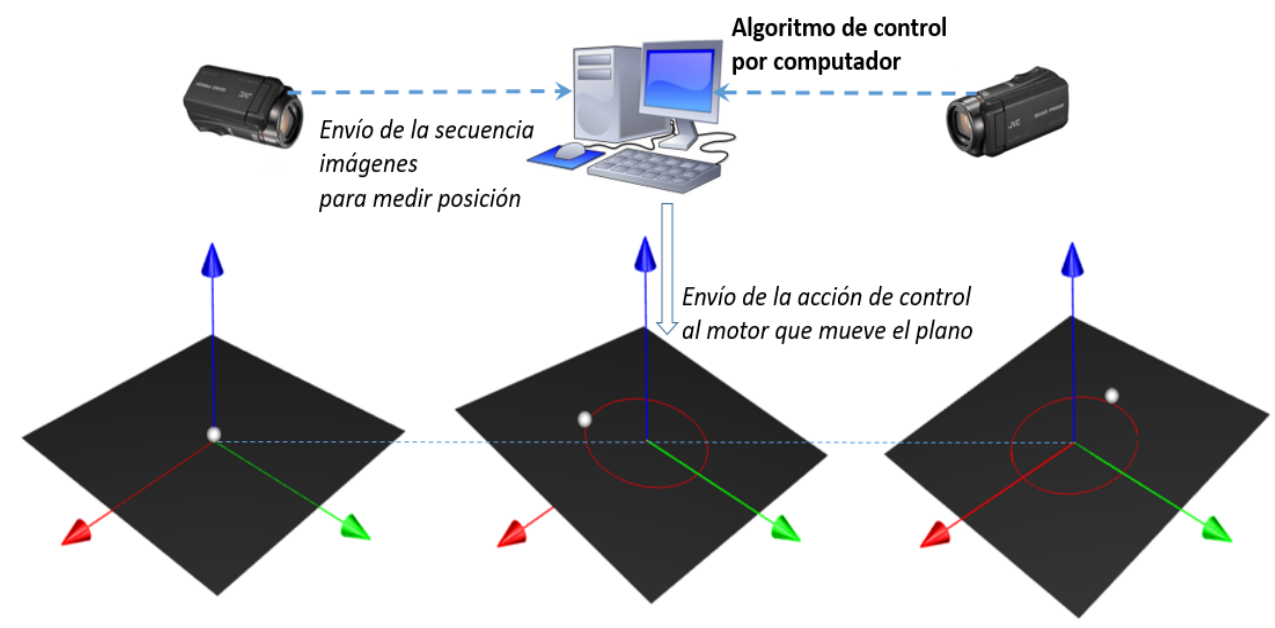

Fig. 3 Control por visión de la trayectoria de una bola deslizante sobre un plano móvil

(cc) EY-NC-ND 2020, Universitat Politècnica de València

Congreso In-Red (2020) 
En la Figura 3 se describe el funcionamiento del bucle de control propuesto. En la parte superior se muestran las cámaras mediante las cuales se mide la posición de la bola sobre el plano, y el ordenador sobre el cual se implementa el algoritmo de control. A su vez, el equipo controlador está conectado a los motores que gobieran el movimiento de los motores que permiten inclinar el plano en ambos ejes, respectivamente (representado con la flecha azul vertical).

En la parte inferior de la figura se puede ver el plano móvil en tres posiciones diferentes. La primera (en la parte izquierda de la figura) muestra la posición de reposo, mientras que las otras dos muestran distintas posiciones de la bola tras fijar como objetivo que describan, por ejemplo, una trayectoria circular.

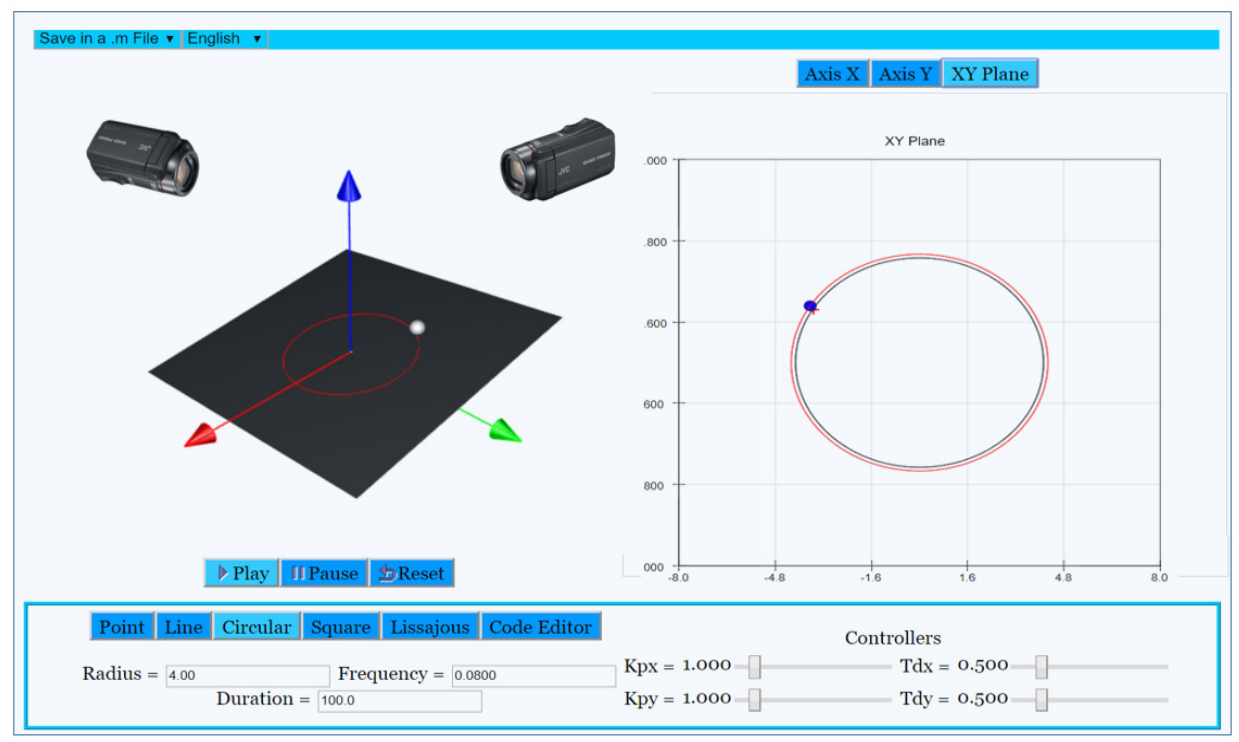

Fig. 4 Laboratorio virtual web para el control de trayectoria de una bola sobre plano móvil.

En la Figura 4 se muestra el interfaz de usuario que aparece tras abrir el fichero HTML con el navegador web. A partir de esta aplicación, los estudiantes podrán visualizar el comportamiento de la planta en función del diseño de controlador que hayan realizado, y cuyos parámetros podrán establecer en el panel inferior para cada uno de los controles en X e Y (parámetros Kpx, Tdx, Kpy, Tdy).

\section{Conclusiones}

En este trabajo se ha presentado una herramienta mediante la cual es posible generar laboratorios virtuales basados en navegadores web y que además pueden operar de forma local en un equipo sin conexión a internet. Las ventajas reseñables de esta propuesta son:

- Permitir que los alumnos puedan realizar las prácticas de la asignatura ante situaciones que les impida asistir físicamente a los laboratorios por causas de fuerza mayor.

- Facilitar a los alumnos la adquisición de las herramientas necesarias para mejorar la comprensión de los contenidos impartidos en el aula.

- Ofrecer la posibilidad de incluir nuevas prácticas con un esfuerzo mínimo, es decir, sin necesidad de que el docente posea conocimientos avanzados en programación.

- Abaratar el coste económico gracias al uso de herramientas estándar que no requieren licencias y la no necesidad de utilizar dispositivos hardware. 
- Garantizar el acceso a los recursos de forma individual independientemente del número total de alumnos por grupo, con la consiguiente escalabilidad de costes.

Además, gracias a la interfaz gráfica compatible con navegadores web, el docente puede fácilmente ilustrar con imágenes y animaciones los ejemplos que se plantean de forma teórica en el aula durante la sesión de problemas. Se ofrece por tanto un valioso recurso que el docente podrá utilizar para facilitar la transmisión de contenidos y asimilación de conceptos.

Finalmente, cabe destacar que, aunque la herramienta propuesta está originalmente enfocada para asignaturas relacionadas con de la ingeniería de control, se puede extender a otras asignaturas de ingeniería que justifiquen su utilización por presentar al mismo tiempo una elevada carga conceptual y una marcada vertiente aplicada. Ante esta situación, el docente debe enfrentarse al desafío de atraer el interés de los estudiantes y mejorar su percepción de la asignatura desde el punto de vista de utilidad práctica de cara a la realidad profesional.

\section{Agradecimientos}

Los autores agradecen el soporte financiero a la Universidad de Zaragoza, proyecto PIIDUZ_19_074 Integración de asignaturas de ingeniería de sistemas y automática dentro de una red nacional de laboratorios interactivos UNILabs para el fomento de las metodologías activas.

\section{Referencias}

BLOOM, B. (1956) Taxonomy of Educational Objectives: The Classification of Educational Goals, New York: Longman.

CHAOS, D., CHACON, J., LOPEZ-OROZCO, J., DORMIDO, S. (2013) "Virtual and remote robotic laboratory using EJS, MATLAB and LabVIEW" en Sensors, vol. 13, issue 2, p. 2595-2612.

CHRISTIAN, W., ESQUEMBRE, F. (2007) "Modeling physics with Easy Java Simulations" en The Physics Teacher, vol. 45, issue 8, p. 475-480.

DORMIDO, R., VARGAS, H., DURO, N., SÁNCHEZ, J., DORMIDO-CANTO, S., FARIAS, G., ESQUEMBRE, F., DORMIDO, S. (2008) "Development of a web-based control laboratory for automation technicians: The threetank system" en IEEE Transactions on Education, vol. 51, issue 1, p. 35-44.

ESQUEMBRE, F. (2004) "Easy Java Simulations: A software tool to create scientific simulations in Java" en Computer physics communications, vol. 156, issue 2, p. 199-204.

FLANAGAN, D. (2006) JavaScript: the definitive guide. Sebastopol: O’Reilly \& Associates.

HACKATHORN, J., SOLOMON, E. D., BLANKMEYER, K. L., TENNIAL, R. E., GARCZYNSKI, A. M. (2011). "Learning by Doing: An Empirical Study of Active Teaching Techniques" en Journal of Effective Teaching, vol. 11, issue 2, p. 40-54.

HERADIO, R., DE LA TORRE, L., GALAN, D., CABRERIZO, F. J., HERRERA-VIEDMA, E., DORMIDO, S. (2016) "Virtual and remote labs in education: A bibliometric analysis" en Computers \& Education, vol. 98, p. 14-38.

HOFF, A. V., SHAIO, S. (1995) Hooked on Java; Creating Hot Web Sites with Java Applets. Boston: AddisonWesley Longman Publishing

JOHNSON, G. W. (1997). LabVIEW graphical programming. US: McGraw-Hill Education. 
LOPEZ, C. P. (2002). Matlab y sus Aplicaciones en las Ciencias y la Ingeniería. Madrid : Pearson Educación.

MARCH, A. F. (2006) "Metodologías activas para la formación de competencias" en Educatio siglo XXI, vol. 24, p. $35-56$.

MICHEL, N., CATER III, JJ, VARELA, O. (2009) “Active versus passive teaching styles: An empirical study of student learning outcomes" en Human resource development quarterly, vol. 20, issue 4, p. 397-418.

SOSNIAK, L. A. (1994). Bloom's taxonomy. Chicago: The University of Chicago Press

TRENSHAW, K. F., REVELO, R. A., EARL, K. A., HERMAN, G. L. (2016) "Using self-determination theory principles to promote engineering students' intrinsic motivation to learn" en International Journal of Engineering Education, vol. 32, issue 3, p. 1194-1207. 